\title{
Resiliencia Psicológica: \\ Una aproximación hacia su conceptualización, enfoques teóricos y relación con el abuso sexual infantil ${ }^{1}$
}

\author{
Psychological resilience: \\ an approach to the concept, theoretical framework and relation with child sexual \\ abuse
}

\author{
Cristián Pinto Cortez ${ }^{1}$ \\ Departamento de Filosofía y Psicología, Universidad de Tarapacá, Arica, Chile
}

(Rec: octubre 2013 - Acep: octubre 2014)

\begin{abstract}
Resumen
El abuso sexual infantil constituye un grave problema de salud pública y una violación reiterada de los derechos humanos de miles de niños, niñas y adolescentes alrededor del mundo. Una prolífera investigación se ha desarrollado al respecto para determinar la magnitud del problema, los efectos psicológicos, los factores de riesgo y de protección. En ese contexto, cobra relevancia el enfoque de resiliencia al explicar los mecanismos que favorecen la adaptación positiva ante la adversidad. En el presente artículo, se expone, en una primera parte, el análisis del concepto de resiliencia y sus diferentes etapas de investigación a través del tiempo. Finalmente, se realiza una integración de este modelo positivo en la comprensión y abordaje de la victimización infanto-juvenil.

Palabras clave: resiliencia, adaptación positiva, adversidad, abuso sexual infantil, factores de protección.
\end{abstract}

\begin{abstract}
Child sexual abuse is a serious public health problem and a violation of human rights from children and adolescents. A prolific research has been developed to determine the magnitude of the problem, psychological effects, risk factors and protective factors. In this context, resilience approach becomes important by explain the mechanisms that promote positive adaptation to adversity. In this paper, it is discussed in the first part, the analysis of the concept of resilience and its various stages of investigation over time. Finally, an integration of this model in understanding and approaching child and adolescent victimization is done
\end{abstract}

Keywords: resilience, positive adaptation, adversity, child sexual abuse, protective factors.

\footnotetext{
Correspondencia dirigida a: Cristián Pinto Cortez. Universidad de Tarapacá, Departamento de Filosofía y Psicología. Dirección postal: 18 de Septiembre \#1191. E-mail: cpinto@estumail.ucm.es.
} 


\section{Introducción}

Los factores protectores son aquellas características personales, familiares o contextuales, que disminuyen las efectos nocivos del estrés sobre la salud y el bienestar (Organización Mundial de la Salud [OMS], 2006). Estos factores, favorecen el desarrollo personal y contrarrestan las consecuencias negativas que se producen en contextos de riesgo (Donas, 1998). Por otra parte, el enfoque de riesgo plantea que, a mayor conocimiento de los eventos vitales negativos, existe mayor probabilidad de actuar sobre ellos de forma anticipada, lo que permite cambiar las condiciones que exponen a las personas o grupos a adquirir enfermedades o sufrir daños (Rutter, 1999).

Para González-Arratia, Valdez, Van Barneveld y González (2012), los factores protectores, al igual que los de riesgo, no actúan de manera aislada sino que ejercen un efecto conjunto. Ambos, establecen complejas relaciones funcionales, de las cuales resulta una atenuación de los efectos negativos ante la adversidad. La comprensión de este proceso de amortiguación del estrés es imprescindible para entender los mecanismos que subyacen a los agentes protectores en general, y a la resiliencia en particular (Rojas, 2007).

Así, las personas resilientes son aquellas que al estar expuestas a un conglomerado de factores de riesgo, tienen la capacidad de utilizar los factores protectores para sobreponerse a la adversidad, crecer y desarrollarse adecuadamente, llegando a madurar como seres adultos competentes, pese a los pronósticos desfavorables.

El objetivo de este artículo, es presentar una síntesis de los principales aportes teóricos citados en la literatura especializadas sobre el concepto de resiliencia. Concretamente, el propósito es aportar a la discusión teórica del término y analizar el rol de este constructo ante las experiencias de victimización sexual infanto-juvenil.

El interés por abordar el abuso sexual infantil dice relación con que éste, es una de las victimizaciones más graves que puede vivir un niño, niña o adolescente y las secuelas pueden ser múltiples y en distintas áreas del desarrollo. En dicho contexto, cobra relevancia incorporar en el estudio del abuso sexual, variables que aporten a la superación de la experiencia traumática.

Para ello, primero se expone una revisión del concepto de resiliencia psicológica individual. En segundo lugar, se repasan las etapas de investigación que se han desarrollando en relación con este enfoque. Por último, se presenta un análisis integrativo entre la teoría de resiliencia y el fenómeno del abuso sexual infantil, apostando por un enfoque positivo en la comprensión y abordaje de las experiencias vitales de niños, niñas y adolescentes afectados por estas graves vulneraciones.

\section{Resiliencia: Revisión del Concepto}

El concepto de resiliencia nace en la Física para referirse a la capacidad de los cuerpos de resistir los impactos y volver a su forma inicial, y ha sido adoptado por las Ciencias Sociales para describir la capacidad de una persona para sobreponerse a una situación adversa. Según el diccionario de la Real Academia Española [RAE], la resiliencia se define como la capacidad humana de asumir con flexibilidad situaciones límite y sobreponerse a ellas.

Sin embargo, el término "resiliencia" también es comúnmente utilizado en textos de Ecología, Medio Ambiente, Microbiología, Regeneración Celular, Procesamiento de Materiales, y diferentes aspectos de la Ingeniería, la Economía y el Marketing Corporativo (Earvolino-Ramírez, 2007). Existen, por lo tanto, definiciones de resiliencia en distintas disciplinas, cada cual con sus propios matices.

En el campo de las ciencias sociales, las relaciones humanas y, en especial, de la Psicología, las definiciones de resiliencia han estado influenciadas por distintos contextos históricos-culturales, y su presencia se ha descrito en el ámbito individual, familiar y comunitario.

Así, la resiliencia familiar se define como la capacidad de la familia para responder de forma positiva a una situación adversa, y salir fortalecida, inclusive, con mayores recursos y mayores niveles de confianza (Simon, Murphy \& Smith, 2005). Por otra parte, la resiliencia comunitaria es la habilidad de los miembros de una comunidad para tomar acciones significativas, deliberadas, y colectivas para resolver el impacto de un problema, incluyendo la capacidad de evaluar el entorno, intervenir y seguir adelante (Pfefferbaum, Reissman, Pfefferbaum, Klomp, Gurwitch, 2005).

En ese contexto, la resiliencia individual se entiende como un proceso mediante el cual las personas se recuperan de la adversidad y pueden salir adelante en sus vidas (Dyery \& McGuinnes, 1996). La resiliencia individual incluye aspectos fisiológicos, emocionales, actitudinales y psicológicos (Resnick, Gwyther \& Roberto, 2011).

Por otra parte, Cabanyes (2010) considera que la resiliencia no representa necesariamente resistencia absoluta sino más bien, el concepto hace referencia a la recuperación e incluye una amplia gama de respuestas 
Tabla 1.

Definiciones de Resiliencia

\begin{abstract}
“La habilidad para recuperarse o enfrentarse exitosamente a pesar de la importancia de la adversidad”(Rutter, 1985, p.599)
"La capacidad de recuperarse de situaciones traumáticas graves"(Cabanyes, 2010, p. 145)

"La resiliencia no es solo una resistencia a la adversidad sino que hace referencia a la capacidad de crecer o desarrollarse en contextos difíciles"(Aldwin, 2007, p. 24).

"La capacidad de una persona para recobrarse fortalecida de la adversidad y dueña de mayores recursos. Se trata de un proceso activo de resistencia, autocorrección y crecimiento como respuesta a las crisis y desafíos de la vida”(Walsh, 2004, p. 4)

"Una capacidad humana universal para hacer frente a las adversidades de la vida, superarlas o incluso ser transformados por ella"(Grotberg, 2001, p. 23)

"La capacidad de los seres humanos sometidos a los efectos de una adversidad, de superarla e incluso salir fortalecidos de la situación”(Cyrulnik, 2003, p. 67)

"Una capacidad universal que permite a una persona, grupo o comunidad impedir, disminuir o superar los efectos nocivos de la adversidad" Vanistendael, 1994, p. 50).

"La adaptación positiva, o la capacidad para mantener o recuperar la salud mental, a pesar de experimentar adversidades "(Herman, Borden, Schultz \& Brooks, 2010, p. 230)

"La capacidad de un niño o niña para desarrollarse bien, para seguir proyectándose en el futuro a pesar de los acontecimientos desestabilizadores, de condiciones de vida difíciles y de traumas a veces graves"(Manciaux, Vanistendael, Lecomte, Cyrulnik, 2003, p. 23).
\end{abstract}

que varían según la naturaleza de las circunstancias adversas.

A lo anterior, se suma la visión de Wald, Taylor y Andersson (2006) quienes definen la resiliencia como la adaptación positiva o a la habilidad de mantener o recuperar la salud mental a pesar de las experiencias adversas.

Asimismo, Ryff y Singer (2003), señalan que las personas resilientes son capaces de mantener su bienestar físico y salud psicológica y tienen la capacidad de recuperarse más rápidamente de eventos estresantes.

Finalmente, para Fletcher y Sarkar (2013), existen dos elementos comunes en las distintas definiciones de resiliencia: adversidad y adaptación positiva (Tabla 1).

Por tanto, un primer paso para aproximarnos al concepto de resiliencia es entender los conceptos de "adversidad"y "adaptación positiva". Respecto a la adversidad, Luthar, Cicchetti y Becker (2000) señalan que normalmente abarca las circunstancias negativas de la vida las cuales estadísticamente están asociadas con dificultades de adaptación. Esta visión es compartida por autores que han investigado las características de la resiliencia en situaciones de desastres naturales, presencia de trauma o sufrimiento humano (Singh, 2006; Singleton, 2004).

Desde otra perspectiva, Davis, Luecken y LemeryChalfant (2009) indican que para la mayoría de las personas, las adversidades no constituyen grandes desastres sino más bien, son dificultades sencillas que están arraigadas en nuestras vidas cotidianas. Según los autores, las adversidades están presenten en contextos familiares, escolares, laborales, entre otros. De acuerdo a esto, Davydov, Stewart, Ritchie y Chaudieu (2010) refieren que los mecanismos de resiliencia pueden ser distintos de acuerdo a la gravedad del contexto, que van desde la resiliencia contra molestias cotidianas habituales como el estrés laboral (adversidad leve) a la resiliencia contra el estrés extremo como en el caso de un duelo o agresión sexual (adversidad severa).

Respecto a la "adaptación positiva", este concepto hace mención a un sistema psicológico de habilidades de afrontamiento utilizadas por las personas expuestas a situaciones traumáticas (Fletcher \& Sarkar, 2013) Este sistema de afrontamiento ante situaciones estresantes permite un ajuste positivo, y el crecimiento y aumento de la capacidad de recuperación emocional. Para Mahoney y Bergman (2002), una adaptación positiva significa la implementación de procesos por los que las personas alcanzan patrones de ajuste inusualmente favorables, teniendo en cuenta sus antecedentes y recursos disponibles. La adaptación positiva es un componente inherente a la definición de resiliencia. 
Ahora bien, la resiliencia es un término que surge desde un enfoque positivo, en contraposición a una visión psicopatologizante del ser humano. Sin embargo, este constructo no ha estado exento de críticas. Por ejemplo, a menudo se reprocha que la resiliencia es un objeto de estudio escurridizo, y que para medirla es importante especificar la escala temporal y espacial; lo resiliente hoy puede no serlo mañana.

Si bien puede quedar relativamente claro el concepto, sobre todo en sus versiones más metafóricas, la operativización del mismo no es tarea sencilla (Reyes \& Ballesteros, 2011). No obstante, hay un acuerdo entre los investigadores respecto de que la resiliencia se puede medir (Grotberg, 2005). En tal sentido, los métodos de estudio apuntan a reconocer y analizar aquellos comportamientos, que construidos en la interacción, operan como factores protectores del daño en un contexto determinado y que conocer y analizar dichos factores contextualmente delimitados, es el objetivo de las diversas propuestas de medición (Villalta, 2010).

Así, un desafío importante para los profesionales en el campo de la investigación y aplicación del enfoque de resiliencia, es la delimitación conceptual del término. Para Luthar, Cicchetti y Becker (2000), es trascendental que los investigadores e investigadoras describan claramente su definición de adversidad y adaptación positiva, justificando razonadamente su uso en el campo de la investigación, ya que para tener utilidad práctica, los conceptos se deben traducir claramente y con precisión. En el caso de la resiliencia, si su conceptualización es ambigua, habrá un conjunto incompleto de definiciones operativas, lo que llevará a una falta de claridad y validez científica.

\section{Etapas en el estudio de la Resiliencia}

Las investigaciones en el campo de la resiliencia han pasado por diversas etapas. Autores como Greene y Conrad (2002) reconocen al menos dos generaciones de estudios en resiliencia, mientras que otros investigadores como Suárez-Ojeda y Melillo (2001) y Richardson (2002) identifican al menos tres, cada uno con un enfoque específico. Más recientemente, O’Dougherty, Masten y Narayan (2013), agregan una cuarta vía de acceso al conocimiento sobre resiliencia humana, incorporando los aportes de las neurociencias. Las cuatro etapas de investigación propuestas por O'Doughtery et al. (2013), servirán de referencia para este trabajo.

\section{Primera generación de estudios}

Los estudios iniciales en el campo de la resiliencia, recibieron una fuerte influencia del entorno sociocultural de los años 70' en los Estados Unidos. En ese contexto, se produjo un cambio de paradigma al cambiar la atención de los factores de riesgo que conducían a los problemas psicosociales hacia la identificación de capacidades y fortalezas en las personas (Benson, 1997). Así, los investigadores de la época, centraron su atención en los factores que convertían a una persona más resiliente que otra, orientando su trabajo a la identificación de ventajas selectivas, tales como el funcionamiento intelectual o las características de personalidad (Zautra, Hall \& Murray, 2010).

A grandes rasgos, es posible señalar que las primeras investigaciones centraron su interés en adversidades que afectaban a niños y niñas, y posteriormente, se incluyeron otras etapas evolutivas como la adolescencia y la adultez.

Según L. Walker (2000 en Walsh, 2004) la primera investigación sobre factores de riesgo y de protección, y que se reconoce como los inicios del estudio de la resiliencia, fue el trabajo de Emy Werner y Ruth Smith, iniciado en 1955 y publicado en 1989. Estas psicólogas dirigieron una investigación longitudinal que incluyó 505 niños y niñas nacidos en 1955 en la isla de Hawai. En el estudio se utilizó un método natural de análisis biográfico, desde el nacimiento de los participantes hasta que tenían aproximadamente 30 años. Los resultados mostraron drásticas diferencias entre quienes habían sido criados en condiciones ambientales similares. Por ejemplo, de los niños y niñas que crecieron en ambientes de pobreza u otras condiciones adversas como divorcio entre los padres, alcoholismo o enfermedad mental, aproximadamente dos tercios desarrollaron serios problemas en la edad adulta, la otra tercera parte se desarrollaron como adultos competentes, confiados, con capacidad para cuidar de otros y de ellos mismos. Es más, los resultados indicaron que el rendimiento académico de este grupo era igual o superior al de los niños de bajo riesgo de la cohorte que habían vivido en ambientes seguros y estables (Walker, 2000 en Walsh, 2004).

En un principio, los investigadores clasificaron a estos niños como "invulnerables", y pensaban que eran inmunes al estrés como consecuencia de su fortaleza interior o su carácter (Anthony, 1974; Pines, 1975 en 
Masten, 2001). Con el tiempo, la investigación avanzó y se amplió el foco de estudio, por lo tanto, el concepto de "invulnerabilidad"fue reemplazado por términos como resistencia al estrés y capacidad de recuperación. Según Werner y Smith (1992), estos conceptos son más adecuados, ya que consideran la resiliencia como un proceso de interacción entre elementos de riesgo y protección.

A esta primera generación de estudios se le reconocen tres aportes importantes:

1. La explicación del efecto acumulativo de factores de riesgo, que indican el "por qué"de la enfermedad y su relación con fenómenos adaptativos en presencia de adversidad (Mejía, 2003).

2. La identificación de factores de adaptación positiva ante cualquier tipo de riesgo, inclusive niveles alto de riesgo. Éstos se conocen como factores compensatorios (Benson, Scales, Leffert \& Roehlkepartain, 1999; Garmezy, Masten \& Tellegen, 1984) ofactores promotores de resiliencia (Sammeroff, 1999).

3. La identificación de factores de adaptación positiva, ante niveles especialmente altos de riesgo, conocidos típicamente como factores protectores (Rutter, 1999).
$\mathrm{Al}$ respecto, el factor compensatorio tiene un efecto directo sobre el resultado, independiente de las consecuencias del factor de riesgo. Por ejemplo, la abstinencia de alcohol es compensatoria en el sentido que se relaciona de forma independiente con un menor riesgo de conductas suicidas en adolescentes (Anderson \& Ledogar, 2008).

Por otra parte, el factor protector modera los efectos que puede ocasionar un factor de riesgo. Los factores de protección influyen de distintas maneras en los resultados de cada persona (Tabla 2). Así, pueden ayudar a neutralizar los efectos de los factores de riesgo, ya que pueden debilitarlos (pero no eliminarlos por completo), o bien pueden mejorar el efecto positivo de otro factor compensatorio (Fleming \& Ledogar, 2008).

\section{Segunda generación de estudios}

La segunda etapa de investigación en resiliencia, aportó datos significativos sobre el proceso que lleva a cabo una persona para adquirir las cualidades resilientes. En palabras de Masten y Morison (1991), las preguntas de investigación de la primera etapa se

Tabla 2.

Factores protectores

\begin{tabular}{ll} 
Autor & Factores protectores \\
\hline Anthony (1974) & Comunicación efectiva, sentido de dignidad personal, sentido de control sobre el destino, \\
& orientación social positiva, asertividad/capacidad de pedir ayuda, redes de apoyo social \\
& informal, habilidades sociales, tolerancia a la frustración, locus de control interno, fle- \\
& xibilidad, percepción de autoeficacia, habilidad para resolver problemas, habilidad para \\
& tomar decisiones, confianza en los demás, sentido del humor, capacidad de pensamiento \\
& crítico, expresión y manejo de emociones, capacidad de adaptación.
\end{tabular}

Benard (1991)

Garmenzy (1991)

Buen carácter, temperamento fácil, relaciones positivas, expectativas altas acerca de si mismo.

Garmenzy (1991)

Masten, Neeman \& Andenas (1994) Buen carácter, relaciones positivas, capacidad para resolver problemas, capacidad para gestionar emociones, pensamiento crítico, sentido de control sobre el destino, confianza en los demás y esperanza futuro.

Rutter (1993)

Werner \& Smith (1992)
Orientación positiva hacia el futuro, uso de talentos para beneficio personal, orientación social positiva, sentido de autoeficacia, sentido del humor, habilidad para tomar decisiones, sensibilidad interpersonal.

Locus de control interno, sentido del humor, habilidad para resolver problemas, pensamiento crítico, asertividad. 
centraron en el "qué", mientras que, en la segunda etapa, en el "cómo".

Este grupo de trabajos, influenciados por el modelo ecológico de Bronffenbrener (1979), plantean un rol preponderante de los sistemas -familias, servicios, grupos y comunidades- frente a la adversidad. Concretamente, la resiliencia es entendida como un proceso dinámico, resultado de la interacción recíproca entre persona y ambiente (Suárez-Ojeda \& Melillo, 2001). Por tanto, se pone énfasis en los recursos ambientales que influyen en el proceso de resiliencia, tales como: acceso a cuidados tempranos, recursos físicos, materiales y acciones profesionales. En este período, los investigadores reconocieron explícitamente la importancia de los sistemas en las explicaciones causales de la resiliencia (Cicchetti, 2010; Cicchetti \& Curtis, 2007; Masten, 2007, 2011). El énfasis está en lo relacional.

Al respecto, Rutter (1992) argumenta que la resiliencia se caracteriza como un conjunto de procesos sociales e intrapsíquicos que posibilitan tener una vida sana, viviendo en un medio insano. Estos procesos tendrían lugar a través del tiempo, dando afortunadas combinaciones entre las características de la persona y su ambiente familiar y social. De esta forma, la resiliencia no puede ser pensada como un atributo con el que los seres humanos nacen, sino que está en desarrollo y en permanente interacción entre la persona y su ambiente (García-Vesga \& Domínguez-De la Ossa, 2013).

En esta segunda etapa de investigaciones, centrada en los aspectos interaccionales, surgen autores como Grotberg, Cyrulnik y Richardson.

Grotberg (1997) aporta el concepto de fuentes interactivas de resiliencia. Según la autora, el ser resiliente no depende sólo de la interacción, sino que además, para hacer frente a las adversidades, las personas toman factores de resiliencia de cuatro fuentes que se visualizan en las expresiones verbales de las personas (niños, adolescentes o adultos) con características resilientes. Estas fuentes se refieren a: "Yo tengo"en mi entorno social, "Yo soy"y "Yo estoy", que hablan de las fortalezas intrapsíquicas y condiciones personales, y "Yo puedo", que concierne a las habilidades en las relaciones con los otros.

En la misma línea, Cyrulnik (2005) refuerza la importancia de la necesidad del otro como punto de apoyo para la superación de la adversidad. Para este autor, la resiliencia surge en la interacción con figuras significativas y se construye en la trayectoria histórica de las personas. A estas figuras claves, las denomina "tutores de resiliencia", que son aquellas personas, instancias, grupos, lugares y acontecimientos que provocan un renacer del desarrollo psicológico tras la adversidad. Para las personas afectadas por situaciones adversas, los tutores de resiliencia son el punto de partida para intentar retomar o iniciar otro tipo de desarrollo en un contexto afectivo y social positivo. Estos tutores pueden ser reales o imaginarios, y actúan como representaciones que promueven confianza y autoimagen positiva.

Richardson (2002), complementa la visión anterior de la resiliencia con su modelo de desafío. Desde este modelo, existe un proceso de "reintegración resiliente" el cual se refiere al aprendizaje positivo que resulta de la experiencia de adversidad. La reintegración resiliente ocurre con el crecimiento tras la adversidad y es el resultado de la identificación de las cualidades resilientes. De acuerdo a esta teoría, las personas están genéticamente predispuestas y poseen un potencial del cual no son conscientes. El proceso de resiliencia es la vía de acceso a esta toma de conciencia.

Finalmente, debido a la importancia del contexto en esta generación de estudios, se desarrollan investigaciones relacionadas con los recursos comunitarios y culturales. Más específicamente, algunos autores como Chandler y Lalonde (1998) acuñan el concepto de resiliencia cultural o continuidad cultural. Estos investigadores de la University British Columbia, usan este concepto para referirse a un atributo de los pueblos originarios que opera para conservar su herencia cultural. Su hipótesis es que las comunidades transmiten a sus jóvenes elementos culturales que promueven la autocontinuidad.

Es más, Hallet, Chandler y Lalonde (2007) en una investigación realizada con pueblos originarios de Canadá, encontraron que las tasas de suicidio se reducían significativamente en las comunidades en las que, al menos, la mitad de los miembros señalaba un conocimiento conversacional de la "lengua nativa".

Este último punto es de suma relevancia para reivindicar las particularidades de cada comunidad, $\mathrm{y}$ al mismo tiempo, reconocer los potenciales de cada cultura y subcultura. Especialmente en nuestro contexto latinoamericano.

\section{Tercera generación de estudio}

El modelo positivo de salud mental y el avance en las prácticas basadas en la evidencia en Psicología, ha determinado que se incorporen cada vez más enfoques centrados en la resiliencia en todo ámbito de intervención (García-Silgo, 2013). Es así como, la tercera etapa de investigación representa una confluencia de 
objetivos, modelos y métodos para la intervención desde el enfoque de resiliencia, junto con el análisis de sus respectivos resultados (Cicchetti, Rappaport, Sandler, \& Weissberg, 2000; Coie et al., 1993; Cowen \& Durlak, 2000; Masten, 2007; Masten \& Coatsworth, 1998; Weissberg \& Kumpfer, 2003). En esta fase, los hallazgos científicos acumulados en la primera y segunda generación de estudios, se aplican de forma práctica a las distintas realidades sociales. A la vez, los resultados de estas intervenciones aportan a reforzar y fortalecer la teoría de la resiliencia. O'Dougthery et al. (2013) la identifican como una etapa de intervención-experimental.

Un ejemplo es el del International Resilience Project (IRP), un proyecto internacional de varios años de duración, liderado por el Resilience Research Centre de la Dalhouise University, y que se ejecuta desde el 2008 con el objetivo de desarrollar una comprensión detallada de los procesos de resiliencia en niños y adolescentes. El IRP utiliza un enfoque intercultural que emplea métodos de investigación cuantitativos y cualitativos para analizar los factores individuales, interpersonales, familiares, comunitarios y culturales asociados a la construcción de la resiliencia en jóvenes de todo el mundo. Además, su propósito es la aplicación de los resultados de la investigación en las poblaciones donde se están realizando los procesos de investigación. Actualmente, el proyecto se ejecuta en Canadá, Estados Unidos, China, Colombia, Gambia, India, Israel, Palestina, Rusia, Sudáfrica y Tanzania.

Los resultados de las aplicaciones prácticas basadas en el enfoque de resiliencia han mostrado resultados satisfactorios (Kraemer, Wilson, Fraibur \& Agras, 2002; Masten, 2011; Weissberg, Kumpfer \& Seligman, 2003). No obstante, la resiliencia no debe confundirse con una técnica de intervención, sino que es un concepto que facilita aplicaciones orientadas a mejorar a las personas, a las familias y en general a las comunidades a enfrentar con éxito situaciones de riesgo.

\section{Cuarta generación de estudios}

La etapa más reciente de investigación en resiliencia se basa en los últimos hallazgos sobre el funcionamiento del cerebro y su relación con el comportamiento humano. Según Feder, Nestler y Charney (2009), en la capacidad de recuperación intervienen factores de tipo ambiental, genéticos, epigenéticos y neuronales, por tanto, la resiliencia estaría mediada por cambios adaptativos cerebrales en distintos neurotransmisores y vías moleculares. Estas modificaciones determinan el funcionamiento de circuitos neuronales que regulan: la gratificación, el miedo, la reactividad emocional y el comportamiento social frente al estrés (Feder et al., 2009).

De acuerdo a Karatsoreos y Mc Ewen (2013), el cerebro detecta los estímulos ambientales, integra esta información en los estados internos, y coordina las respuestas fisiológicas y conductuales más apropiadas.

En la misma línea, Paulus et al. (2010), señalan que una respuesta óptima ante condiciones adversas requiere de dos elementos importantes: (1) la capacidad de procesamiento neuronal y (2) las capacidades cognitivas y de aprendizaje para la adaptación en ambientes extremos. En este contexto, las dos áreas del cerebro responsable de dichos procesos son la corteza insular y la amígdala. Un buen funcionamiento de estas estructuras cerebrales es un factor relevante en la respuesta resiliente.

Para Paulus et al. (2010), el enfoque de la resiliencia desde las neurociencias tiene una ventaja sobre los modelos descriptivos tradicionales debido a que:

1. Una vez identificado el rol de los sustratos neuronales ${ }^{1}$, se pueden realizar intervenciones sobre ellos. 2. El estudio de los sustratos neuronales implicados en las respuestas a ambientes adversos, pueden determinar los procesos afectivos y cognitivos necesarios para una modulación óptima de la respuesta resiliente.

Graham (2010) relaciona la teoría de la resiliencia con la teoría de la neurobiología interpersonal de Siegel, destacando la importancia del contexto y las relaciones interpersonales en el desarrollo del cerebro y la mente infantil. Siegel (2007) sugiere algunos principios básicos y los ingredientes esenciales de la experiencia que facilitan el desarrollo de la mente, el bienestar y la resiliencia psicológica. Por ejemplo: los cuidados tempranos, el buen trato, la capacidad de sintonizar emocionalmente (cuidador-niño/a), la empatía y la capacidad del calmar el estrés en el niño(a), son elementos fundamentales para que el cerebro infantil se desarrolle de forma plena. De esta manera, se produce la permanencia de las tareas esperadas para cada etapa del desarrollo y se fortalecen los recursos personales, a nivel cognitivo, emocional, social y conductual, necesarios para gestionar la adversidad (Siegel, 2012; Siegel, 2007).

La neurobiología interpersonal define el bienestar mental en términos de una integración entre las

\footnotetext{
Conjunto de estructuras cerebrales que subyacen a comportamientos específicos o estados psicológicos.
} 
relaciones interpersonales, la mente y el cerebro. La integración se define en términos de los procesos opuestos de diferenciación y vinculación. Por ejemplo, cuando áreas separadas del cerebro se especializan en las funciones que les corresponden y se vinculan entre sí, decimos que el sistema está integrado y saludable. La integración promueve que el sistema funcione como una unidad con las características de flexibilidad, adaptación, coherencia, vigor y estabilidad. Este flujo coherente de información está delimitado por los extremos del caos y la rigidez, parámetros que permiten diagnosticar desbalances mentales. Por lo tanto, el bienestar mental proviene del desarrollo de relaciones empáticas, una mente coherente y un cerebro integrado (Siegel, 2012; Siegel, 2007). En síntesis, la integración es el proceso básico que toda relación afectiva segura facilita, al promover el bienestar psicológico (Graham, 2010).

Aunque la descripción total de estos importantes descubrimientos están fuera del alcance de este capítulo, es importante destacar que, en la perspectiva de la resiliencia basada en las neurociencias, confluyen una integración de modelos e investigación sobre ecosistemas, sistemas sociales, biología individual y el sistema nervioso (Longstaff, 2009; Masten \& Obradovic, 2008; Norris et al., 2008), constituyéndose como un enfoque complementario a las fases anteriores de investigación.

Esta etapa de investigación recién comienza, pero promete transformar la aplicación práctica de la resiliencia.

\section{La relación entre Abuso Sexual Infantil (ASI) y Resiliencia}

La investigación en el campo del abuso sexual ha prestado poca atención a las víctimas que presentan una disminución de efectos psicopatológicos y sociales, vale decir, a las más resilientes.

Sin embargo, dado el impacto negativo de una agresión sexual en el desarrollo psicológico infantil, la capacidad de recuperación es fundamental para que niños, niñas y adolescentes estén en condiciones óptimas para superar futuras dificultades. En ese contexto, el enfoque de resiliencia en el estudio y abordaje del abuso sexual, es un aporte trascendental para mejorar la calidad de vida de las víctimas (Kumpfer, 1999).

Como primer punto, es necesario aclarar que no necesariamente toda experiencia de abuso sexual produce graves daños psicológicos y sociales en las víctimas. Hay evidencia suficiente que avala la existencia de niños y adolescentes que presentan muy pocos síntomas.
López-Sánchez (1994), establece que entre un 20\% y un $30 \%$ de las víctimas de abuso sexual infantil permanecen estables emocionalmente tras la agresión. Otros presentan "efectos durmientes", es decir, en un principio no muestran síntomas (no necesariamente por ausencia de daño), sino que los efectos del abuso aparecen inclusive, hasta un año después de ocurrida la agresión. Es más, Widom (1999) señala que estos efectos pueden aparecer hasta en la edad adulta, debido a una revictimización o incluso en ausencia de ésta. Un suceso estresante o que recuerde el abuso sufrido puede hacer aparecer la sintomatología.

Ahora bien, estas diferencias individuales observadas en niños y niñas víctimas de abuso sexual, ha sido el punto de partida para que los investigadores se interesaran en la capacidad de recuperación tras la agresión. Así, con una tendencia similar a las etapas de investigación en resiliencia, los primeros trabajos en el campo del abuso sexual se centraron casi exclusivamente en los efectos negativos a nivel psicopatológico y social, posteriormente se incluyen estudios orientados a identificar factores de riesgo y de protección (Finkelhor, 1986; López-Sánchez, 1994).

\section{Efectos Psicológicos del Abuso Sexual Infantil}

Respecto a los efectos psicológicos del trauma por abuso sexual, los especialistas han identificado la existencia de un conjunto de síntomas que afectan a las víctimas. A corto plazo se han reconocido síntomas característicos (aunque no diagnósticos) como: conducta sexualizada (Beitchman et al., 1991; Putnam, 2003), trastorno por estrés pos-traumático (Trask, Walsh \& DiLillo, 2011), reacciones ansioso-depresivas (Echeburúa \& De Corral, 2006), fracaso escolar, dificultades inespecíficas de socialización y comportamiento sexualmente agresivo (Cantón \& Cortés 2001; Echeburúa \& De Corral, 2006), entre otros.

Autores como Fontanella, Harrington y Zuravin (2000), han establecido la relación entre psicopatología y género, señalando que los niños son más proclives a experimentar síntomas de tipo externalizante (comportamiento agresivo, dificultades de socialización) mientras que, las niñas, de tipo internalizantes (reacciones ansioso-depresivas). Sin embargo, en estudios recientes queda de manifiesto que esta relación no está del todo clara (véase Hillberg, Hamilton-Giachritsis \& Dixon, 2011; Maikovich-Fongy \& Jaffee, 2010).

Por otra parte, los efectos a largo plazo del abuso sexual (a partir de dos años siguientes a la experiencia de abuso) han sido considerados especulativos, destacando 
la dificultad que entraña su estudio, especialmente al ser comparado con los efectos a corto plazo, y principalmente dada su interacción con otro tipo de factores relacionados con el paso del tiempo (López-Sánchez, 1993 en Pereda, 2010; Noguerol, 1997).

En ese contexto, se han descrito las siguientes consecuencias psicológicas a largo plazo: insatisfacción sexual (Guimond, 2008), promiscuidad (Spiegel, 2008), aumento del riesgo de victimización (Disch, 2006). La ideación o conducta suicida también parecen ser más comunes en las víctimas de abuso sexual (Beitchman, et al., 1991; Putnam, 2003). Además, se ha descrito la relación con otros cuadros psicopatológicos como depresión (Thomas, DiLillo, Walsh \& Polusny, 2011), trastorno por estrés pos traumático (Guerrero, Torres \& Conde, 2012), trastorno límite de personalidad (Machizawa-Summers, 2007), trastorno antisocial de la personalidad (Semiz, Cengiz, Servet \& Mesut, 2007) y consumo de drogas y alcohol (Bebbington et al., 2011).

\section{Factores de Riesgo del Abuso Sexual Infantil}

Se han identificado un número significativo de factores de riesgo del abuso sexual infantil. Finkelhor, Hotaling, Lewis y Smith (1990), señalan que son agentes de riesgo: el haber crecido con un solo padre (principalmente con la madre) o con ninguno de ellos, en un clima familiar conflictivo y haber recibido escasa o nula educación sexual.

Por otra parte Putnam (2003), en su trabajo denominado "Ten-Research Update Review: Child Sexual Abuse"en donde sistematizó más de una década de trabajos sobre ASI, identificó los siguientes factores de riesgo: sexo - las mujeres tienen más probabilidad de ser agredidas sexualmente que los hombres-, la edad - a partir de los 12 años y durante la adolescencia existe mayor probabilidad de ser víctima de abuso sexual-, la discapacidad física y/o psíquica, nivel socioeconómico - el nivel socioeconómico bajo es un factor de riesgo de maltrato físico y abuso sexual-, la estructura familiar- la ausencia de uno o ambos padres aumenta la probabilidad de sufrir abuso al igual que la presencia de un padrastro aumenta la probabilidad de abuso para las niñas-, respecto a la dinámica familiar, son determinantes de riesgo: discapacidad de los padres, madres particularmente enfermas, madres con problemas de alcoholismo, ausencia permanente de la figura materna, conflictos de pareja severos, consumo de drogas por parte de los padres, aislamiento social, crianza basada en el castigo, familias con historias de abusos transgeneracionales.
En general, los estudios posteriores al trabajo de Putnam, aportan datos similares en cuanto a identificación de factores de riesgo, evidenciando poca variación. Por ejemplo, Pinto (2013) señala que el hacinamiento y la co-ocurrencia de otros tipos de malos tratos en la infancia son factores de riesgo del abuso sexual infantil. Por su parte, Butler (2013) señala nuevamente como factor de riesgo, la ausencia de uno o ambos padres, un nivel de educación bajo de la madre, bajo nivel socioeconómico, poca calidez en la relación madre-hijo(a), y agrega como factores de riesgo, características psicológicas del niño (a) como presencia de síntomas internalizantes y externalizantes (previos al abuso sexual), bajo rendimiento escolar y necesidades educativas especiales.

\section{Factores Protectores, Resiliencia y Abuso Sexual Infantil}

Debido a la magnitud del problema, el abuso sexual es una amenaza real para cualquier niño, niña o adolescente; sin embargo, se han descrito ciertas características personales y en la interacción con la familia que son consideradas factores de protección, y que son importantes de fortalecer en el proceso de crianza. Éstos se contextualizan como factores compensatorios o promotores de resiliencia. Rispens, Aleman y Goudena (1997) evaluaron la eficacia de 16 programas de prevención del abuso sexual en el ámbito escolar, e identificaron los siguientes factores compensatorios: enseñar a los niños(as) conceptos sobre abuso sexual, habilidades de auto-protección y acceso a recursos económicos y sociales. Putnam (2003) señala que el entrenamiento en competencias parentales a familias insertas en contextos de riesgo, es un factor protector del abuso sexual y de la negligencia. Para Cardozo y Dubini (2006), la existencia de soportes y recursos externos aparece como uno de los pilares de la promoción de resiliencia, es decir: la familia, los vínculos de amistad y la escuela. Para Finkelhor (2009), son elementos importantes de prevención del abuso sexual: enseñar a los niños habilidades para identificar situaciones de riesgo, el fortalecimiento de competencias personales como capacidad de comunicar las cosas que están ocurriendo, así como pedir ayuda.

No obstante, un vez ocurrida la agresión y tras el sufrimiento, también existen factores que posibilitan la recuperación y la adaptación positiva de niños, niñas y adolescentes. Así, con los resultados de las investigaciones sobre resiliencia, el interés respecto 
a esclarecer las variables que amortiguan los efectos psicológicos y sociales del abuso sexual impulsó lo que se conoce como los estudios de factores protectores (Jessor, 1992).

Si bien en un principio el interés estuvo centrado en variables mediadoras que significaban un riesgo en el incremento del daño asociado al abuso, como también a las que lo disminuían, un número importante de investigaciones se ha centrado en los factores de protección propiamente tal.

Por ejemplo, Spaccarelli (1994) refiere que existen variables en relación con la víctima, en relación con el entorno familiar y social, en relación con las características del abuso sexual, y en relación con el agresor, las cuales mediarían las consecuencias psicosociales del abuso. Spaccarelli y Kim (1995) defienden que aquellas víctimas de abuso sexual infantil resilientes son las que presentan un mayor nivel de apoyo parental (post develación), y un menor nivel de eventos negativos posteriores al abuso (conflictos parentales, múltiples entrevistas con profesionales diferentes, entre otros.)

En esa línea, Mrazek y Mrazek (1987) encontraron en los niños(as) maltratados(as) sexualmente, destrezas personales como capacidad para reconocer el peligro, adaptación, capacidad de distanciarse de sentimientos intensos, crear redes de apoyo, y ubicarse a sí mismo en el tiempo y en el espacio proyectándose a futuro libre de la imagen de quienes le causaron daño.

Morrow y Smith (1995) en una investigación con víctimas de abuso sexual, describieron un modelo especifico de estrategias cognitivas y emocionales. En él identificaron habilidades resilientes como la gestión activa de sentimientos de impotencia y locus de control interno. A esto se sumó, la capacidad de resistir ante sentimientos amenazantes y peligrosos generados por el abuso.

En otra investigación, Singleton (2004) examinó la resiliencia en mujeres afroamericanas víctimas de ASI, identificando estrategias de resiliencia con una orientación colectiva, como por ejemplo, creencias espirituales asociadas a un orden divino y conexiones con fuertes modelos femeninos.

Los elementos que identificó Singleton (2004), coincidieron con los resultados de Morrow y Smith (1995), al identificar el locus de control interno positivo como estrategia resiliente. Es decir, un aumento de la conciencia en las víctimas de que los estados emocionales dependen de sí mismo, y no de las circunstancias.

Recientemente, Marriott, Hamilton-Giachritsis, y Harrop (2013) revisaron 50 artículos científicos (19912010), y en éstos identificaron una serie de factores asociados con niveles altos de resiliencia frente al abuso sexual infantil. Estos incluyen recursos internos, como, por ejemplo, habilidades de afrontamiento, interpretación de experiencias y autoestima. Además, se consideran recursos de la comunidad, como conexión con redes sociales, la iglesia o el colegio.

En la Tabla 3 se describen en detalle las relaciones entre los distintos elementos descritos hasta ahora, y que hacen referencia a la relación entre abuso sexual infantil y resiliencia.

Como ha quedado en evidencia, las características individuales de la víctima, y los recursos que cuente en su contexto sociofamiliar y social, son fundamentales en el proceso de recuperación. Así, se han descrito factores promotores de la resiliencia que pueden aportar a prevenir situaciones de abuso sexual, y en los casos en que ha ocurrido, potenciar factores protectores a nivel individual, familiar y social que impulsen la recuperación y el bienestar general. En ese contexto, desde el enfoque de resiliencia visualizaremos al niño, niña o adolescente, con sus propias potencialidades y recursos, los cuales pueden ser o no desarrollados según los distintos contextos a los que se vea expuesto. La vivencia de abuso sexual, evidentemente, no determinará su vida, ya que el niño (a) tendrá otras oportunidades para vivenciar el buen trato o el potenciamiento de sus recursos resilientes. Y es ahí, precisamente, donde cumplen un rol fundamental, los profesionales que se dedican a la intervención psicológica y social en casos de abusos sexual infantil.

\section{Conclusión}

La resiliencia es un constructo teórico que ha sido objeto de diversos estudios e investigaciones, y hoy en día, es innegable el aporte de este enfoque a las ciencias sociales en general, y a la psicología en particular. Sin embargo, en un principio, desde el contexto académico se realizaron diversas críticas, que inclusive, pusieron en duda el valor científico del término. Parece ser, que la aplicación excesiva del término, se convirtió en ocasiones, en un claro abuso conceptual (Becoña, 2006) o en una frase cliché, que tuvo la ventaja de ser bien recibido, tanto en el ámbito científico como en los medios masivos (Gómez, Valenzuela \& Sotomayor, 2012). Más aún, Tisseron (2009) en Gómez et al. (2012) advierte a sus lectores sobre los malentendidos que puede implicar la aplicación de este concepto: se corre el riesgo de una postura moralista y subestimar 
RELACIÓN CON EL ABUSO SEXUAL INFANTIL

Tabla 3.

Relación entre Resiliencia y Abuso Sexual Infantil

\begin{tabular}{|c|c|c|}
\hline Termino & Definición & Ejemplo \\
\hline Adversidad & $\begin{array}{l}\text { Alteraciones en la función de un sistema; } \\
\text { experiencias que amenazan la adapta- } \\
\text { ción o desarrollo }\end{array}$ & $\begin{array}{l}\text { Abuso sexual infantil en todas sus formas y tipología intra- } \\
\text { familiar, extra familiar, con contacto (tocaciones, violación, } \\
\text { obligar al niño a realizar conductas sexuales) o sin contacto } \\
\text { (exhibicionismo, reproducción de material pornográfico, uti- } \\
\text { lización de niños (as) en pornografía, etc.). Grooming online. }\end{array}$ \\
\hline Resiliencia & $\begin{array}{l}\text { Adaptación positiva frente al riesgo o } \\
\text { adversidad, capacidad de un sistema } \\
\text { dinámico para resistir o recuperarse de } \\
\text { las amenazas }\end{array}$ & $\begin{array}{l}\text { Niño o niña que a pesar de las vivencias de abuso sexual, } \\
\text { mantiene un adecuado rendimiento académico y social (tiene } \\
\text { amigos, se lleva bien con el profesor) en el colegio. En general, } \\
\text { no vea afectadas sus áreas de desarrollo. }\end{array}$ \\
\hline Riesgo & $\begin{array}{l}\text { Alta probabilidad de resultados } \\
\text { negativos. }\end{array}$ & $\begin{array}{l}\text { El abuso sexual conlleva un riesgo significativo de desarrollos } \\
\text { de problemas psicopatológicos y sociales. }\end{array}$ \\
\hline Factor de riesgo & $\begin{array}{l}\text { Una característica en un grupo de per- } \\
\text { sonas o situaciones que predicen un } \\
\text { resultado negativo. }\end{array}$ & $\begin{array}{l}\text { Edad, pobreza, discapacidad intelectual, hacinamiento, vio- } \\
\text { lencia intrafamiliar aumenta la probabilidad de sufrir abuso } \\
\text { sexual. }\end{array}$ \\
\hline Riesgo acumulativo & $\begin{array}{l}\text { Aumento del riesgo debido a la presencia } \\
\text { de múltiples factores de riesgo. }\end{array}$ & $\begin{array}{l}\text { Niños (as) que se han desarrollado en contextos de deprivación } \\
\text { socioeconómica, que además han sido abandonados e insertos } \\
\text { en sistemas de protección. }\end{array}$ \\
\hline Vulnerabilidad & $\begin{array}{l}\text { Capacidad disminuida de una persona o } \\
\text { un grupo de personas para anticiparse, } \\
\text { hacer frente y resistir a los efectos de un } \\
\text { peligro natural o causado por la actividad } \\
\text { humana, y para recuperarse de los mis- } \\
\text { mos. Es un concepto relativo y dinámico }\end{array}$ & $\begin{array}{l}\text { Características de personalidad de niños y niñas, como sobre } \\
\text { adaptación, timidez, ansiedad, dificultades de comunicación. }\end{array}$ \\
\hline Riesgo próximo & $\begin{array}{l}\text { Factores de riesgo experimentados di- } \\
\text { rectamente por el niño (a) }\end{array}$ & $\begin{array}{l}\text { Ser testigo de violencia, ser víctima de otros tipos de malos } \\
\text { tratos. }\end{array}$ \\
\hline Riesgo distante & $\begin{array}{l}\text { Riesgos derivados del contexto ecológi- } \\
\text { co de un niño, pero que esta mediado por } \\
\text { procesos proximales }\end{array}$ & Vivir en comunidades con altas tasas de criminalidad. \\
\hline $\begin{array}{l}\text { Factores } \\
\text { promotores o } \\
\text { compensatorios }\end{array}$ & $\begin{array}{l}\text { Una característica en un grupo de } \\
\text { personas o situación que predice } \\
\text { un resultado positivo o deseable, de } \\
\text { manera similar a los niveles altos y } \\
\text { bajos de riesgo. }\end{array}$ & $\begin{array}{l}\text { Padres con habilidades parentales adecuadas; acceso } \\
\text { a recursos económicos y sociales. Conexión con redes } \\
\text { comunitarias iglesia, grupos deportivos, escuela, etc. }\end{array}$ \\
\hline $\begin{array}{l}\text { Factores } \\
\text { protectores }\end{array}$ & $\begin{array}{l}\text { Un predictor de resultados positivos } \\
\text { especialmente en situaciones de riesgo } \\
\text { o la adversidad }\end{array}$ & $\begin{array}{l}\text { Locus de control interno positivo, gestión activa de emo- } \\
\text { ciones y sentimientos, autoestima, inteligencia, capacidad } \\
\text { de distanciarse de la imagen de quienes han causado daños } \\
\text { interpretación de experiencias, capacidad de crear redes de } \\
\text { apoyo, credibilidad y apoyo post-develación. }\end{array}$ \\
\hline
\end{tabular}


Protección acumulativa La presencia de múltiples factores protectores en la vida de una persona
Un niño niña en un barrio con altos niveles de criminalidad, tiene un hogar seguro, apoyo de un profesor de la escuela, y las relaciones positivas con compañeros, la comunidad o distintas organizaciones

Relaciones positivas con compañeros y profesores. Éxito en el rendimiento escolar. Desarrollo de la capacidad cognitiva y de aprendizaje.

esperadas según la etapa del desarrollo.

Tareas del

Hitos psicosociales o logros previstos desarrollo para las personas en diferentes edades en un contexto histórico y cultural determinado, estos criterios sirven para valorar la adaptación de la persona.
Caminar, hablar, aprender a leer;

Hacer amigos; adaptarse a normas y límites; empatía y comportamiento prosocial, aprender idiomas, música, resolver problemas. la relevancia de los traumas y las reacciones que ante ellos puede tener el ser humano. Así, otras críticas se han erigido en torno a:

1.- La inexistencia de un conjunto definitivo de elementos que constituyan factores de riesgo o de protección (Hoge, Austin \& Pollack, 2007). Actualmente, éstos pueden ser cualquier variable que muestre un aumento o una disminución de resultados positivos y negativos.

2.- El escaso desarrollo de medidas de resiliencia, que dificultan la comparación y generalización de resultados (Friborg et al., 2005).

3.- Vaguedad del término (Gordon \& Song, 1994).

Sin embargo, el concepto de resiliencia ha hecho gala de su propio proceso de resistencia frente a las críticas. Hoy en día este enfoque tiene un gran alcance, y se ha demostrado empíricamente que la resiliencia tiene un poder explicativo en la adaptación exitosa de muchas personas que han vivido traumas o eventos vitales negativos (Becoña, 2006).

Si bien, y como ha quedado de manifiesto en este artículo, las críticas iniciales al concepto de resiliencia han sido subsanadas en gran medida, aún queda mucho por hacer. Especialmente, en lo referente el desarrollo de instrumentos, el análisis de la resiliencia en distintos contextos culturales, y la integración de los aportes de las neurociencias.

Respecto a la resiliencia y el abuso sexual infantil, lo primero que hemos de decir, es que el enfoque de resiliencia es significativamente útil en la teoría y práctica de la psicoterapia y la intervención psicosocial con víctimas de abuso sexual. En primer lugar, facilita el descubrimiento de los factores que protegen a las personas frente a la adversidad, y por tanto, permiten intervenir en ellos, disminuyendo los resultados negativos. En segundo lugar, favorece una visión positiva de las personas afectadas por el abuso sexual, aportando a un entendimiento de las víctimas al margen del daño. Es más, el enfoque de resiliencia, ha confirmado la premisa de que el abuso sexual no determina la vida, y que es posible un desarrollo adecuado y una calidad de vida mejor, a pesar de haber vivido estas graves vulneraciones.

Ahora bien, en Latinoamérica en general y en Chile en particular, se han realizado importantes aportaciones al estudio, sistematización y práctica de la resiliencia (Kotliarenco, Cáceres \& Fontecilla, 1997; Suárez-Ojeda \& Melillo, 2001). Sin embargo, es escaso el tratamiento otorgado en este mismo ámbito a la aplicación de la resiliencia al campo del abuso sexual infanto-juvenil. Al término de este trabajo, no se encontró ningún estudio publicado en revistas especializadas sobre teoría y/o práctica de la resiliencia en el trabajo con víctimas de abuso sexual realizado en nuestro contexto nacional.

Sin embargo, la inexistencia de trabajos realizados y publicados en revistas ad hoc, no implica que múltiples profesionales adscritos a la corriente de la psicología positiva, estén incorporando en sus prácticas terapéuticas y psicoterapéuticas el enfoque de resiliencia. Por tanto, surge como necesidad, la sistematización y comunicación de experiencias locales en el trabajo con víctimas de abuso sexual desde el enfoque de resiliencia, y asimismo, aumentar la difusión de resultados de trabajos e investigaciones.

En ese contexto, es una tarea pendiente desarrollar medidas de resiliencia en nuestro contexto cultural, así como, considerar el rol que ejerce la cultura en 
la construcción de la resiliencia. Especialmente, en nuestro territorio impregnado por la diversidad cultural.

En resumen, se puede afirmar que el estudio de la resiliencia es un campo de investigación relativamente joven, y como tal, han surgido todo tipo de debates, tanto a favor como en contra. Pese a esto, el estudio de la resiliencia tiene un apoyo empírico suficiente para ampliar líneas de investigación, y en consecuencia, apoyar la presencia de factores que posibiliten la recuperabilidad de niños, niñas, adolescentes y adultos afectados por la lacra del abuso sexual.

\section{Referencias}

Aldwin, C. (2007). Stress, coping, and development: An integrative perspective. New York: Guilford.

Andersson, N. \& Ledogar, R. J. (2008). The CIET Aboriginal youth resilience studies: 14 years of capacity building and methods development in Canada. Pimatisiwin: Journal of Aboriginal and Indigenous Community Health, 6(2), 65-88. Recuperado de http://www.ncbi.nlm.nih.gov/pmc/articles/PMC2942846/pdf/ nihms1051.pdf

Anthony, E. J. (1974). The syndrome of the psychologically invulnerable child. En E. Anthony \& C. Koupernik (Eds.) The child in his family: Children at psychiatric risk (pp. 529-545). New York: Wiley.

Bebbington, P., Jonas, S., Kuipers, E., King, M., Cooper, C., Brugha, T. \& Jenkins, R. (2011). Childhood sexual abuse and psychosis: data from a cross-sectional national psychiatric survey in England. The British Journal of Psychiatry, 199(1), 29-37. doi:10.1192/ bjp.bp. 110.083642

Becoña, E. (2006). Resiliencia: definición, características y utilidad del concepto. Revista de psicopatología y psicología clínica, 11(3), 125-146. Recuperado de http://e-spacio.uned.es/fez/ eserv/bibliuned:Psicopat-2006-E3EEEFE3-E4DF-43B4-C15DFF038F693092/Documento.pdf

Beitchman, J., Zucker, K., Hood, J., Da Costa, G. \& Akman, D. (1991). A review of the short-term effects of child sexual abuse. Child abuse \& neglect, 15(4), 537-556. doi:10.1016/01452134(91)90038-F

Benard, B. (1991). Fostering resiliency in kids: Protective factors in the family, school, and community. Portland. Northwest Regional Educational Laboratory.

Benson, P. (1997). All kids are our kids. Minneapolis: Search Institute.

Benson, P. L., Scales, P. C., Leffert, N. \& Roehlkepartain, E. (1999). A fragile foundation: The state of developmental assets among American youth. Minneapolis: Search Institute.

Bronfenbrenner, U. (1979). Contexts of child rearing: Problems and prospects. American Psychologist, 34(10), 844-850. doi:10.1037/0003-066X.34.10.844

Butler, A. C. (2013). Child sexual assault: Risk factors for girls. Child abuse \& neglect, 37(9), 643-652. doi:10.1016/j. chiabu.2013.06.009

Cabanyes, J. (2010). Resiliencia: una aproximación al concepto. Revista de Psiquiatría y Salud Mental, 3(4), 145-151. doi:10.1016/j. rpsm.2010.09.003

Cantón, J. \& Cortés, M. R. (2001). Sintomatología, evaluación y tratamiento del abuso sexual infantil. En V. E. Caballo \& M. A.
Simón (Eds.), Manual de psicología clínica infantil y del adolescente. (pp. 293-321). Madrid: Pirámide

Cardozo, G. \& Dubini, P. (2006). Promoción de salud y resiliencia en adolescentes desde el ámbito escolar. Psicología, Cultura y Sociedad, 7, 21-39. Recuperado de http://www.palermo.edu/ cienciassociales/publicaciones/pdf/Psico7/7Psico\%2002.pdf

Chandler, M. \& Lalonde, C. (1998). Cultural continuity as a hedge against suicide in Canada's First Nations. Transcultural Psychiatry, 35(2), 191-219. doi:10.1177/136346159803500202

Cicchetti, D. (2010). Resilience under conditions of extreme stress: A multilevel perspective. World Psychiatry, 9, 1-10. doi:10.1002/j.2051-5545.2010.tb00297.x

Cicchetti, D., \& Curtis, W. J. (2007). Multilevel perspectives on pathways to resilient functioning. Development and Psychopathology, 19(3), 627-629. doi:10.1017/S0954579407000314

Cicchetti, E., Rappaport., J, Sandler, I. \& Weissberg, R. (2000). The promotion of wellness in children and adolescents. Child Welfare League of America.

Coie, J., Watt, N., West, S., Hawkins, J., Asarnow, J., Markman, H., ... \& Long, B. (1993). The science of prevention: A conceptual framework and some directions for a national research program. American Psychologist, 48, 1013-1022. doi:10.1037/0003066X.48.10.1013

Cowen, E. \& Durlak, J. (2000). Social policy and prevention in mental health. Development and Psychopathology, 12(4), 815-834. Recuperado de http://journals.cambridge.org/article_S0954579400004132

Cyrulnik, B. (2003). El murmullo de los fantasmas: volver a la vida después de un trauma. Barcelona: Gedisa.

Cyrulnik, B. (2005). Bajo el signo del vínculo. Madrid: Gedisa.

Davis, M., Luecken, L., \& Lemery-Chalfant, K. (2009). Resilience in common life: Introduction to the special issue. Journal of personality, 77(6), 1637-1644. doi: doi:10.1111/j.14676494.2009.00595.x

Davydov, D., Stewart, R., Ritchie, K. \& Chaudieu, I. (2010). Resilience and mental health. Clinical Psychology Review, 30(5), 479-495. doi:10.1016/j.cpr.2010.03.003

Disch, E. (2006). Sexual Victimization and Revictimization of Women by Professionals: Client Experiences and Implications for Subsequent Treatment. Women \& Therapy, 29(12), 41-61. doi:10.1300/J015v29n01_03

Donas, S. (1998). Protección, riesgo y vulnerabilidad. Sus posibles aplicaciones en la promoción, prevención, tratamiento y rehabilitación de la salud integral de los/as adolescentes. Segunda versión preliminar. Caracas: Organización Panamericana de la Salud, Organización Mundial de la Salud.

Dyery, J. \& McGuiness, M. (1996). Resilience: Analysis of the concept. Archives of Psychiatric Nursing, vol. 10, 276-282. doi:10.1016/S0883-9417(96)80036-7

Earvolino-Ramírez, M. (2007). Resilience: A concept analysis. Nursing Forum, 42(2), 73-82. doi:10.1111/j.1744-6198.2007.00070.x

Echeburúa, E. \& Corral, P. (2006). Secuelas emocionales en víctimas de abuso sexual en la infancia. Cuadernos de Medicina Forense, 12(43-44), 75-82. Recuperado de http://scielo.isciii.es/ $\mathrm{pdf} / \mathrm{cmf} / \mathrm{n} 43-44 / 06 . p d f$

Feder, A., Nestler, E. \& Charney, D. (2009). Psychobiology and molecular genetics of resilience. Nature Reviews Neuroscience, 10(6), 446-457. doi:10.1038/nrn2649

Finkelhor, D. (2009). The prevention of childhood sexual abuse. The future of children, 19(2), 169-194. doi:10.1353/foc.0.0035

Finkelhor, D. (1986). A sourcebook on child sexual abuse. Beverly Hills: Sage Publications.

Finkelhor, D., Hotaling, G., Lewis, I. \& Smith, C. (1990). Sexual abuse in a national survey of adult men and women: Prevalence, 
characteristics, and risk factors. Child abuse \& neglect, 14(1), 19-28. doi:10.1016/0145-2134(90)90077-7

Fleming, J. \& Ledogar, R. J. (2008). Resilience and indigenous spirituality: a literature review. Pimatisiwin, 6(2), 47-64. Recuperado de http://www.pimatisiwin.com/uploads/404389036.pdf

Fletcher, D. \& Sarkar, M., (2013). A review of Psychological Resilience. European Psychologist, 18(1), 12-23. doi:10.1027/10169040/a000124

Fontanella, C., Harrington, D. \& Zuravin, S. J. (2000). Gender differences in the characteristics and outcomes of sexually abused preschoolers. Journal of Child Sexual Abuse, 9(2), 21-40. doi:10.1300/J070v09n02_02

Friborg, O., Barlaug, D., Martinussen, M., Rosenvinge, J. \& Hjemdal, O. (2005). Resilience in relation to personality and intelligence. International journal of methods in psychiatric research, 14(1), 29-42. doi:10.1002/mpr.15

García-Silgo, M. (2013). Revisión de programas de resiliencia basados en la evidencia en los ejércitos. Sanidad Militar, 69(3), 182-194. doi:10.4321/S1887-85712013000300005

García-Vesga, M. \& Domínguez-De la Ossa, E. (2013). Desarrollo teórico de la Resiliencia y su aplicación en situaciones adversas: Una revisión analítica. Revista Latinoamericana de Ciencias Sociales, Niñez y Juventud, 11(1), 63-77. Recuperado de http://revistaumanizales.cinde.org.co/index.php/Revista-Latinoamericana/ article/view/833

Garmezy, N. (1991). Resilience in children's adaptation to negative life events and stressed environments. Pediatric Annals, 20(9), 459-463. doi:10.3928/0090-4481-19910901-05

Garmezy, N., Masten, A. \& Tellegen, A. (1984). The study of stress and competence in children: A building block for developmental psychopathology. Child Development, 55(1), 97-111. doi:10.2307/1129837

Gómez, G., Valenzuela, F. \& Sotomayor, C. (2012). Jóvenes chilenos de condición vulnerable y rendimiento destacado en comprensión lectora participantes en OECD-PISA 2009 [Documento de Trabajo $\mathrm{N}^{\circ}$ 3]. Centro de Investigación Avanzada en Educación, Universidad de Chile, Santiago. Recuperado de http://www.ciae.uchile.cl/ download.php?file=doctrabajo/03-102012.pdf

González-Arratia, N., Valdez J., Van Barneveld, H. \& González, S. (2012). Resiliencia y factores protectores en menores infractores y en situación de calle. Psicología y Salud, 22(1), 49-62. Recuperado de http://revistas.uv.mx/index.php/psicysalud/article/view/557

Gordon, E. \& Song, L. (1994). Variations in the experience of resilience. En E. Gordon \& L. Song (Eds.), Educational resilience in inner-city America: Challenges and prospects (pp. 27-43). New York: Routledge.

Graham, L. (2010). The Neuroscience of Resilience. The Whise Brain Bulletin, 4(6), 1-15. Recuperado de http://lindagraham-mft.net/ pdf/WiseBrainBulletin-4-6.pdf

Greene, R. \& Conrad, A. (2002). Basic assumptions and terms. En R. Greene (Ed.), Resiliency: An integrated approach to practice, policy, and research (pp. 29-62). Washington DC. NASW Press.

Grotberg, E. (1997). The international resilience project. En M. Jhon (Ed.), A charge against society: the child's right to protection (pp.19-32). London: Jessica Kingsley Publishers.

Grotberg, E. (2001). Nuevas tendencias en Resiliencia. Resiliencia: Descubriendo las propias fortalezas. Buenos Aires: Paidós

Grotberg, E. (2005, Julio). Resilience for tomorrow. Trabajo presentado en la International Council of Psychologists Convention, Foz do Iguaçu, Brazil. Recuperado de http://resilnet.uiuc.edu/library/ grotberg2005_resilience-for-tomorrow-brazil.pdf

Guerrero N., Torres. R. \& Conde, C. (2012). Preliminary study of cerebral proteomics expression of hippocampal region from rats exposed to different stress levels induced by forced swimming. Revista de la Universidad Industrial de Santander. Salud, 44(1),
17-27. Recuperado de http://revistas.uis.edu.co/index.php/revistasaluduis/article/view/2734

Guimond, J. (2008). Childhood sexual abuse and adult sexuality. Dissertation Abstracts International: Section B: The Sciences and Engineering, 68(8-B), 2008. 5572.

Hallett, D., Chandler, M. \& Lalonde, C. (2007). Aboriginal language knowledge and youth suicide. Cognitive Development, 22(3), 392-399. doi:10.1016/j.cogdev.2007.02.001

Herman, K., Borden, L. A., Schultz, T. \& Brooks, C. (2010). The Incredible Years Parent Training Program: Promoting resilience through evidence-based prevention groups. Group Dynamics: Theory, Research, and Practice, 14(3), 230-241. doi:10.1037/ a0020322

Hillberg, T., Hamilton-Giachritsis, C. \& Dixon, L. (2011). Review of meta-analyses on the association between child sexual abuse and adult mental health difficulties: A systematic approach. Trauma, Violence, \& Abuse, 12(1), 38-49. doi:10.1177/1524838010386812

Hoge, E., Austin, E. \& Pollack, M. (2007). Resilience: Research evidence and conceptual considerations for posttraumatic stress disorder. Depression and Anxiety, 24(2), 139-152. Recuperado de http://www.iirp.edu/article_detail.php?article_id=NDc5

Jessor, R. (1992). Risk behavior in adolescence: A psychosocial framework for understanding and action. Developmental Review, 12, 374-390. doi:10.1016/1054-139X(91)90007-K

Karatsoreos, I. \& Mc Ewen, B. (2013). Resilience and vulnerability: a neurobiological perspective. F1000prime reports, 5(13), 1-5. doi:10.12703/P5-13

Kotliarenco, M., Cáceres, I. \& Fontecilla, M. (1997). Estado de arte en resiliencia. Washington DC: Organización Panamericana de la Salud.

Kraemer, H., Wilson, G., Fairburn, C. \& Agras, W. (2002). Mediators and moderators of treatment effects in randomized clinical trials. Archives of General Psychiatry, 59, 877-883. doi:10.1001/ archpsyc.59.10.877

Kumpfer, K. (1999). Factor and processes contributing to resilience: the resilience framework. En M. Glantz \& J. Johnson. (Eds.), Resilience and development: positive life adaptations (pp. 179-224). New York: Kluwer/Plenum.

Longstaff, P. (2009). Managing surprises in complex systems: Multidisciplinary perspectives on resilience. Ecology and Society, 14(1), 49-50. Recuperado de http://www.ecologyandsociety.org/ vol14/iss $1 /$ art49/

López-Sánchez, F. (1994). Abuso Sexual a Menores; Lo que recuerdan de mayores. Madrid: Centro de Publicaciones, Ministerios de Asuntos Sociales.

Luthar, S., Cicchetti, D. \& Becker, B. (2000). The construct of resilience: Implications for interventions and social policies. Development and psychopathology, 12(4), 857-885. Recuperado de http://www.ncbi.nlm.nih.gov/pmc/articles/PMC1903337/pdf/ nihms21560.pdf

Machizawa-Summers, S. (2007). Childhood trauma and parental bonding among Japanese female patients with borderline personality disorder. International Journal of Psychology, 42(4), 265-273. doi:10.1080/00207590601109276

Mahoney, J. \& Bergman, L. R. (2002). Conceptual and methodological considerations in a developmental approach to the study of positive adaptation. Journal of Applied Developmental Psychology, 23(2), 195-217.

Maikovich-Fong, A. \& Jaffee, S. (2010). Sex differences in childhood sexual abuse characteristics and victims' emotional and behavioral problems: Findings from a national sample of youth. Child Abuse \& Neglect, 34(6), 429-437. doi:10.1016/j.chiabu.2009

Manciaux, M., Vanistendael, S., Lecomte, J. \& Cyrulnik, B. (2003). La resiliencia: resistir y rehacerse. Madrid: Gedisa. 
Marriott, C., Hamilton-Giachritsis, C. \& Harrop, C. (2013). Factors promoting resilience following childhood sexual abuse: a structured, narrative review of the literature. Child Abuse Review., 23(1), 17-34. doi:10.1002/car.2258

Masten A. \& Morison, P. (1991). Peer reputation in middle childhood as a predictor of adaptation in adolescence: A 7-year follow-up. Child Development, 62(5), 991-1007. doi:10.1111/j.1467-8624.1991.tb01585.x

Masten, A. (2007). Resilience in developing systems: Progress and promise as the fourth wave rises. Development and psychopathology, 19(03), 921-930. doi:10.1017/S0954579407000442

Masten, A. (2011). Resilience in children threatened by extreme adversity: Frameworks for research, practice, and translational synergy. Development and Psychopathology, 23, 141-154. doi:10.1017/S0954579411000198

Masten, A. \& Obradovic, J. (2008). Disaster preparation and recovery: Lessons from research on resilience in human development. Ecology and Society, 13(1), 1-9. Recuperado de http:// www.ecologyandsociety.org/vol13/iss1/art9/

Masten, A. \& Coatsworth, J. (1998). The development of competence in favorable and unfavorable environments: Lessons from research on successful children. American psychologist, 53(2), 205-220. doi:10.1037/0003-066X.53.2.205

Masten, A., Neemann, J. \& Andenas, S. (1994). Life events and adjustment in adolescents: The significance of event independence, desirability, and chronicity. Journal of Research on Adolescence, 4(1), 71-97. doi:10.1207/s15327795jra0401_5

Mejía, R. (2003). Resiliencia: Ilusión o realidad. CES Medicina, 17(1), 57-62. Recuperado de http://revistas.ces.edu.co/index. php/medicina/article/view/518

Morrow, S. \& Smith, M. (1995). Constructions of survival and coping by women who have survived childhood sexual abuse. Journal of Counseling Psychology, 42(1), 24-33. doi:10.1037/00220167.42.1.24

Mrazek, P. \& Mrazek, D. (1987). Resilience in child maltreatment victims: A conceptual exploration. Child Abuse \& Neglect, 11(3), 357-366. doi:10.1016/0145-2134(87)90009-3

Noguerol, V. (1997). Aspectos psicológicos del abuso sexual infantil. En J. Casado, J. A. Díaz \& C. Martínez (Eds.), Niños maltratados (pp. 177-182). Madrid: Díaz de Santos.

Norris, F., Stevens, S., Pfefferbaum, B., Wyche, K. \& Pfefferbaum, R. (2008). Community resilience as a metaphor, theory, set of capacities, and strategy for disaster readiness. American journal of community psychology, 41(1-2), 127-150. doi:10.1007/s10464$007-9156-6$

O’Dougherty W., Masten, A. S. \& Narayan, A. J. (2013). Resilience processes in development: Four waves of research on positive adaptation in the context of adversity. En S. Goldstein \& R. Brooks (Eds.), Handbook of Resilience in Children (pp. 15-37). New York: Springer.

Organización Mundial de la Salud [OMS] (2006). Revista Panamericana de Salud Pública. Ginebra Suiza.

Paulus, M. Simmons, E., Potterat, K., Van Orden K. \& Swain, J. (2010). Systems Neuroscience approaches to measure brain mechanisms underlying resilience -towards optimizing performance. En S. Komguth, R. Steinberg \& M. Matthews (Eds.), Physiological Factors During High-Tempo Operations (pp. 133-145). United States: Ashgate.

Pereda, N. (2010). Consecuencias psicológicas a largo plazo del abuso sexual infantil. Papeles del psicólogo, 31(2), 191-201. Recuperado de http://pepsic.bvsalud.org/pdf/eureka/v9n1/a07.pdf

Pfefferbaum, B., Reissman, D., Pfefferbaum, R., Klomp, R. \& Gurwitch, R. (2005). Building resilience to mass trauma events. En L. Doll, S. Bonzes, J. Mercy \& D. Sleet (Eds.), Handbook on injury and violence prevention interventions (pp. 347-358). New York: Kluwer Academic Publishers.

Pinto, C. (2013). Prevalencia del abuso sexual masculino en el extremo norte de Chile: secuelas a largo plazo, factores de protección y de riesgo (Tesis doctoral). Universidad Complutense de Madrid, España.

Putnam, F. W. (2003). Ten-year research updates review: Child sexual abuse. Journal of the American Academy of Child \& Adolescent Psychiatry, 42(3), 269-278. doi:10.1097/00004583200303000-00006

Resnick, B., Gwyther, L. \& Roberto, K. (2011). Resilience in aging: concepts, research, and outcomes. United States: Springer.

Reyes, J. \& Ballesteros, E. (2011). Resiliencia Socioecológica: aportaciones y retos desde la Antropología. Revista de Antropología Social, 20, 109-135. Recuperado de http://revistas.ucm.es/index. php/RASO/article/view/36264

Richardson, G. (2002). The metatheory of resilience and resiliency. Journal of clinical psychology, 58(3), 307-321. doi: 10.1002/ jclp. 10020

Rispens, J., Aleman, A. \& Goudena, P. (1997). Prevention of child sexual abuse victimization: a meta-analysis of school programs. Child Abuse \& Neglect, 21(10), 975-987. doi:10.1016/ S0145-2134(97)00058-6

Rutter, M. (1985). Resilience in the face of adversity. British journal of psychiatry, 147(1), 598-611. doi:10.1192/bjp.147.6.598

Rutter, M. (1992). Factors and processes contributing to resilience. En K. Kumpfer. Resilience and development (pp. 179-224). United States: Springer.

Rutter, M. (1993). Resilience: Some conceptual considerations. Journal of adolescent health, 14(8), 626-631. doi:10.1016/1054139X(93)90196-V

Rutter, M. (1999). Resilience concepts and findings: implications for family therapy. Journal of family therapy, 21(2), 119-144. doi:10.1111/1467-6427.00108

Ryff, C. \& Singer, B. (2003). Flourishing under fire: Resilience as prototype of challenge thriving. En C. Keyes \& J. Haidt (Eds.), Flourishing: Positive and the life well-lived. (pp. 15-36). Washington: American Psychological Association.

Sammeroff, A. (1999). Ecological perspectives on development risk. En D. Osofsky \& H. Fitzgerald (Eds.), WAIMH handbook of infant mental health (pp. 223-248). New York: Wiley.

Semiz, U., Cengiz., B, Servet, E. \& Mesut, C. (2007). Childhood trauma history and dissociative experiences among Turkish men diagnosed with antisocial personality disorder. Society Psychiatry Epidemiology, 42(11), 865-873. doi:10.1007/s00127-007-0248-2

Siegel, D. (2007). La mente en desarrollo. Cómo interactúan las relaciones y el cerebro para modelar nuestro ser. Bilbao: Desclée de Brouwer.

Siegel, D. J. (2012). El cerebro del niño. España: ALBA Editorial.

Simon, J., Murphy, J. \& Smith, S. (2005). Understanding and fostering family resilience. The Family Journal, 13(4), 427-436. doi:10.1177/1066480705278724

Singh, A. (2006). Resilience Strategies of South Asian Women who have Survived Child Sexual Abuse (Tesis doctoral). Universidad Estatal de Georgia, Estados Unidos.

Singleton, K. (2004). Strategies of survival: Coping, characteristics of resiliency, and perceived impact of abuse in resilient Black female survivors of sexual trauma. Dissertation Abstracts International Section B: The Sciences \& Engineering, 64(1).

Spaccarelli, S. (1994). Stress, appraisal, and coping in child sexual abuse: A theoretical and empirical review. Psychological bulletin, 116(2), 340-362. doi:10.1037/0033-2909.116.2.340

Spaccarelli, S. \& Kim, S. (1995). Resilience criteria and factors associated with resilience in sexually abused girls. Child abuse \& neglect, 19(9), 1171-1182. doi:10.1016/0145-2134(95)00077-L 
Spiegel, J. (2008). Sexual self-concept and sexually abused males: Early data on the development of a scale. International Journal of Sexual Health, 20(3), 147-161. doi:10.1080/19317610802239990

Suárez-Ojeda, E. \& Melillo, A. (2001). Resiliencia. Descubriendo las propias fuerzas. Buenos Aires: Paidós.

Thomas, R., DiLillo, D., Walsh, K. \& Polusny, M. A. (2011). Pathways from child sexual abuse to adult depression: The role of parental socialization of emotions and alexithymia. Psychology of Violence, 1(2), 121-135. doi:10.1037/a0022469

Trask, E. V., Walsh, K. \& DiLillo, D. (2011). Treatment effects for common outcomes of child sexual abuse: A current meta-analysis. Aggression and violent behavior, 16(1), 6-19. doi:10.1016/j. avb. 2010.10.001

Vanistendael, S. (1994). Cómo crecer superando los percances: resiliencia capitalizar las fuerzas del individuo. Ginebra: International Catholic Child Bureau.

Villalta, M. (2010). Factores de resiliencia asociados al rendimiento académico en estudiantes de contextos de alta vulnerabilidad social. Revista de Pedagogía, 31(88), 159-190. Recuperado de http://saber.ucv.ve/ojs/index.php/rev_ped/article/view/649

Wald, J., Taylor, S. \& Andersson, G. (2006). Literature review of concepts: Psychological resilience. Toronto $(\mathrm{ON})$ : Defence R\&D.

Walsh, F. (2004). The concept of family resilience: Crisis and challenge. Family process, 35(3), 261-281. doi:10.1111/j.15455300.1996.00261x

Weissberg, R., Kumpfer, K. \& Seligman, M. (2003). Prevention that works for children and youth: An introduction. American Psychologist, 58, 425-432. doi:10.1037/0003-066X.58.6-7.425

Werner, E. \& Smith, R. (1992). Overcoming the odds: High risk children from birth to adulthood. New York: Cornell University Press.

Widom, C. (1999). Posttraumatic stress disorder in abused and neglected Children grown up. American Journal of Orthopsychiatry, 56, 1223-1229. doi:10.1176/ajp.156.8.1223

Zautra, A., Hall, J. \& Murray, K. (2010). Handbook of adult resilience. United States: Guilford Press. 